\title{
Fuzzy System Inference and Fuzzy Cognitive Maps for a Cognitive Tutor of Algebra
}

\author{
Pedroza-Mendez Blanca-Estela ${ }^{1}$, Reyes-García Carlos-Alberto ${ }^{2}$, \\ González-Calleros Juan Manuel $^{3}$, Guerrero-García Josefina ${ }^{3}$ \\ 1,Tecnológico Nacional de México, Instituto Tecnológico de Apizaco, Apizaco, \\ Tlaxcala, Mexico \\ ${ }^{2}$ Instituto Nacional de Astrofísica, Óptica y Electrónica (INAOEP), Puebla, Mexico \\ ${ }^{3}$ Benemérita Universidad Autónoma de Puebla, Puebla, Mexico \\ blanca.pmeapizaco.tecnm.mx, kargaxxi@inaoep.mx, \\ \{jumagoca, joseguga\}@gmail.com
}

\begin{abstract}
A cognitive tutor is an intelligent tutorial focused on supporting students on the resolution of problems. The large majority of the architectures for tutorial systems consider three common and indispensable modules: the student module; the tutor module; and, the domain module. These three modules are in charge of executing part of the more important activities within the tutor, and it is within these where it is necessary to implement models that simulate decision taking the way a human expert would. The student module should be constantly evaluating the progress made by students; the tutor module should provide adequate content, based on both the student's characteristics and the learning goals; and, the domain module should be capable of simulating the knowledge of experts regarding teaching-learning methodologies. Somehow, these three modules should interact in order to achieve the goals of the cognitive tutor. In this paper a proposal of fuzzy logic-based models for each of these modules is presented; the design of a system in which the three modules interact is also shown.
\end{abstract}

Keywords: fuzzy cognitive maps, cognitive tutor, problems resolution.

\section{Introduction}

Intelligent Tutoring System (ITS) is a system capable to guide students along a particular domain of knowledge through the solving of tasks tailored to the needs of the student [2]. In turn, a cognitive tutor is a type of ITS with a long-time proven efficacy. Its efficacy is based on its capacity to provide individualized support for the learning of complex cognitive abilities through the practice of problem solving [8]. According to Gonzalez [2], the key components of traditional ITS can be organized in different modules as a student model, domain model, tutor model and interface or communication module, which interact with the user. In this paper, the interest is focused on the three first modules, which contain the representation of expert knowledge in areas related to evaluation processes, teaching and learning methodologies and the detection of cognitive skills of students. These activities have 
the purpose of guaranteeing that the implemented educational designs are adequate for learning achievement. These designs are teaching processes whose implementation isn't easy without help from automatized tools such as intelligent tutoring systems. For this research in particular we are focusing on systems for algebra teaching through problems solving, using fuzzy cognitive maps, for represent the mental schemas that model dependencies between key concepts of domain, in this case algebra, and a fuzzy model for the detection of students' expertise level.

Since the student model module requires the implementation of strategies for the detection of students' cognitive skills for problem resolution in the chosen knowledge field (algebra), it was necessary, first, to use an instrument for the constant measurement of such skills. The instrument is based on the one proposed in [11], known as the 3UV (3 uses of the variables) model. This instrument was adapted in order to associate it with Bloom's Taxonomy, which is a cognitive method for educational objectives whose educational goals were classified as six cognitive levels: remember, understand, apply, analyze, evaluate and create [9]. The instrument based on 3UV model, are the rubric of input to the fuzzy inference model (equation 8). The goal of the inference system is to classify the student with values that are not very rigid and that may provide information that is closer to reality than a rigid model would.

However, in order to develop more efficient tools, not only should knowledge representation be considered as a base. One should go in depth into something more complex known as the representation of dependency between key knowledge concepts in a given domain. This is supported by different pedagogical theories such as the Cognitive Load Theory, which computationally can be modeled through Fuzzy Cognitive Maps (FCM). A FCM helps in the decision making process and allow us to represent such dependencies. This tool is in an initial testing phase with students. Therefore, the results presented in this paper are experimental and are related, execution-wise, to map efficiency. For the tutor module, a fuzzy inference model was also considered; this model allows, based on the results of the student and domain models, the determination of the kind of problems on which the student should conduct further work. For its part, the Cognitive Load Theory (CLT), is based on the assumption that the construction and automatization of cognitive schemas for learning are the main goals of teaching. But those objectives may be thwarted by the limited capacity of working memory. Due to this factor, the proper allocation of available cognitive resources is essential for the learning process [4]. Here lies the importance of the representation of the dependency between the key concepts in the knowledge domain to be learned, and of not only using a general representation of knowledge. In other words, FCM must represent how the knowledge of a domain concept of the teaching material, may be affected by the knowledge of another domain concept [1].

\section{Basic Concepts}

\subsection{Modeling Fuzzy Cognitive Maps}

Fuzzy Cognitive Map (FCM) is fuzzy-graph structure for representing causal reasoning, analyze inference patterns and they act as a nonlinear dynamical system [5]. In education, the causality characteristic allows the FCMs to be adequate to represent 
the dependence between key concepts of some domain of the knowledge in question, allowing to detect the learning material that should be delivered, to some student, with respect to their knowledge level and personal needs $[1,10]$.

For FCM reasoning process, a simple mathematical formulation is usually used. A model implication converges to a global stability, equilibrium in the state of the system. During the inference process, the sequence of patterns reveals the inference model. The mathematical representation of FCMs has the following form $[3,5,6]$ :

$$
A_{i}(k+1)=f\left(A_{i}(k)+\sum_{j=1}^{N} A_{i}(k) \cdot e_{j i}\right)
$$

where $\mathrm{f}(\cdot)$ is a threshold (activation) function. Sigmoid threshold function gives values of concepts in the range $[0,1]$ and its mathematical type is:

$$
f(x)=\frac{1}{1+e^{-m \cdot x}},
$$

where $m$ is a real positive number and $\mathrm{x}$ is the value $\mathrm{A}^{(\mathrm{k})}{ }_{\mathrm{i}}$ on the equilibrium point.

\subsection{Fuzzy Cognitive Maps in Education}

However, when working with the causal dependency between the knowledge levels that a student possesses on a particular domain subject, a situation may arise in which the increase in the concept of any of the nodes of the fuzzy cognitive map may not be total, as represented in equations 1 and 2. Because of this, in these cases we should use a model that helps determine the causality between the nodes based on the dependency of the knowledge domain of the learning material. Chrysafiady et al. [1] define such model as a tuple $(\mathrm{C}, \mathrm{W}, \mathrm{KL}, \mathrm{f})$, where:

1. $C=\left\{C_{1}, C_{2}, \ldots C_{n}\right\}$ is the set of concepts of the domain knowledge.

2. $\mathrm{W}:\left(\mathrm{C}_{\mathrm{i}}, \mathrm{C}_{\mathrm{j}}\right) \rightarrow \mathrm{W}_{\mathrm{ij}}$ is a connection matrix, where $\mathrm{w}_{\mathrm{ij}}$ is a weight of the directed ard from $C_{i}$ to $C_{j}$, which denotes that the knowledge level of the concept $C_{i}$ affects that of concept $\mathrm{C}_{\mathrm{j}}$.

3. $\mathrm{KL}$ is a function that at each concept $\mathrm{C}_{\mathrm{i}}$ associates the sequence of its activation degree. In other worlds, $\mathrm{KL}_{\mathrm{i}}(\mathrm{t})$ indicates the value of a concept's knowledge level at the moment $\mathrm{t}$.

4. $\mathrm{f}$ is a transformation function. For the definition of the transformation function the following limitation has to be taken into account. Only the knowledge level of the most recently read concept affects the knowledge level of a domain concept, each time. Consequently, the KL value of a concept is affected only by the KL value of the most recently read concept, regarding the weight of the directed arc that connects them. Therefore, the transformation function for a FCM, which is used to represent the domain knowledge of the learning material, is defined as:

$$
\mathrm{KL}_{\mathrm{i}}(\mathrm{t}+1)=\mathrm{f}\left(\mathrm{KL}_{\mathrm{i}}(\mathrm{t}) \pm \mathrm{w}_{\mathrm{ji}} * \mathrm{p}_{\mathrm{j}} * \mathrm{KL}_{\mathrm{i}}(\mathrm{t}) / 100\right),
$$


where $p_{j}$ is the percentage of the difference on the value of the knowledge level of the most recently read concept $\mathrm{C}_{\mathrm{j}}$, with $\mathrm{p}_{\mathrm{i}}=\left(\mathrm{KL}_{\mathrm{j}}(\mathrm{t}+1)-\mathrm{KL}_{\mathrm{j}}(\mathrm{t})\right)^{*} 100 / \mathrm{KL}_{\mathrm{j}}(\mathrm{t})$. Also, the $+\mathrm{is}$ used in case of increase and the - is used in case of decrease.

\subsection{Analytical Model for evaluation of learning}

The areas and Cognitive ability is closely related to learning acquisition and is the basis for developing instructional strategies, and their preferences are the basis for developing the type of mentoring. Rongmei proposes an analytical model, combined with fuzzy logic to somehow categorize students based on their cognitive ability and propose a model that is able to make the appropriate instructional design decision to the student, based on their progress. The evaluation model can be expressed via a triple, such as formula [7].

$$
\mathrm{M}=(\mathrm{U}, \mathrm{V}, \mathrm{A}),
$$

where, $\mathrm{U}=(\mathrm{u} 1, \mathrm{u} 2, \mathrm{u} 3, \mathrm{u} 4, \mathrm{u} 5, \mathrm{u} 6)$, are the weight of each of the six points of knowledge of Bloom's Taxonomy, which is given by the experts. V= (v1, v2, v3, v4, v5), these elements respectively stand for five reviews: excellent, good, medium, passed and fail, which is based on both the results of cognitive ability synthetic evaluation and the test.

A is a matrix where each line has the following form:

$$
A_{i}=\left(a_{1}, a_{2}, a_{3}, a_{4}, a_{5}, a_{6}\right)
$$

where $a_{i}$ are values between 0 and 1 , and are define as:

$$
a_{i}=\frac{r_{i j}(1)}{r_{i j}(1)+r_{i j}(0)+r_{i j}(-1)^{\prime}}
$$

$\mathrm{r}_{\mathrm{ij}}(1)$ is defined as the number of correct answers of the student in each of the cognitive abilities, $r_{i j}(0)$ is the number of unanswered questions and $r_{i j}(-1)$ is the number of incorrect answers in each of the cognitive skills.

Finally, $\mathrm{M}$ is defined as:

$$
M=\sum_{i=1}^{6} g_{i} * u_{i}
$$

where

$$
G=W \cdot A=\left(g_{1}, g_{2}, g_{3}, g_{4}, g_{5}, g_{6}\right)
$$

and $\mathrm{W}$ defines the weight of each rubric, this is:

$$
W=\left(w_{1}, w_{2}, w_{3}, w_{4}, w_{5}, \ldots w_{n}\right) .
$$




\section{Description of the Proposed Models}

Concretely, the designed domain module for the cognitive tutor is in charge of analyzing the dependencies between the topics that are considered as the main goal in the learning-teaching process, algebra, for this case. For practical purposes, the syllabus for the subject of algebra of a public, higher secondary level educational system in Mexico was chosen. This syllabus is divided into three units; for the modeling of dependencies between the topics, a fuzzy cognitive map for each unit was built. To obtain the values for the edges of each FCM, a survey with 18 higher secondary level math teachers was used. In Figure 3 the FCM of first unit is showed.

Generally, the processes of the three models in which this work is focused can be seen as the iterative process shown in Figure 1. The first process is the one related with the student model module; process 2 is the domain module; and, process 3 is the one that executes the activities of the tutor model module. The iterative process starts with the handling of rubrics based on the 3UV model, which are evaluated through the analytical model described in the section 2.3 so, using this model, the input parameters for the first FIS are obtained. The model for process 1 is a Fuzzy Inference System (FIS) type Mamdami whose parameters are shown in Figure 2, and it uses as input parameters the levels of Bloom's Taxonomy, which work as the fuzzy sets for the FIS. The fuzzy value obtained of process 1 is used how input to the process 2 .

The second process is a model of fuzzy cognitive maps, whose goal is to identify the relationships between the concepts of the knowledge domain (algebra) in order to determine its relationship with the students' cognitive skill and, based on these relationships, obtain the input parameters for the second FIS, which determines the problems that should be presented to the student in order to help him(her) improve his(her) problem solving skills. For the iterative process for each FCM and for getting the convergence parameters, the models of sections 2.1 and 2.2 are applied. The relationship between these three processes gives way to an iterative process that constantly relates the results of each of the former ones; so in process 1 , a condition is established that gives guidelines to the execution of the three processes, as long as the cognitive evaluation doesn't reach adequate values for each of the three defined fuzzy cognitive maps.

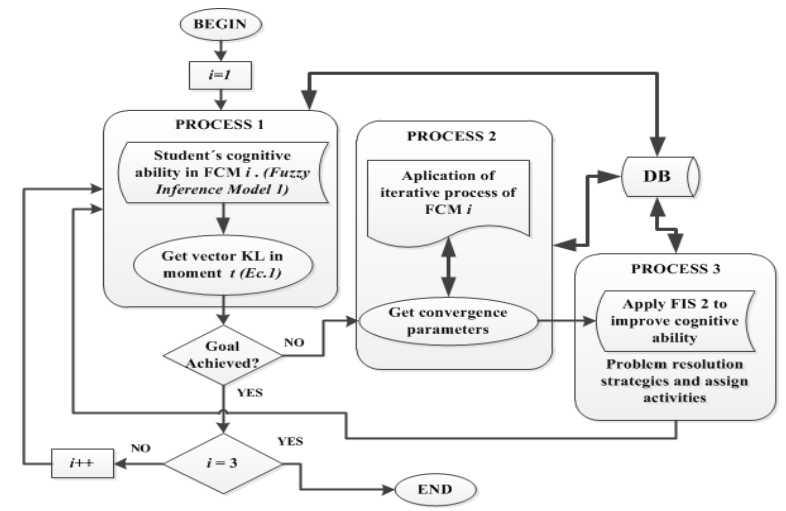

Fig. 1. Iterative process for the three modules of cognitive tutor. 
Blanca-Estela Pedroza-Mendez, Carlos-Alberto Reyes-García, Juan Manuel González-Calleros, et al.

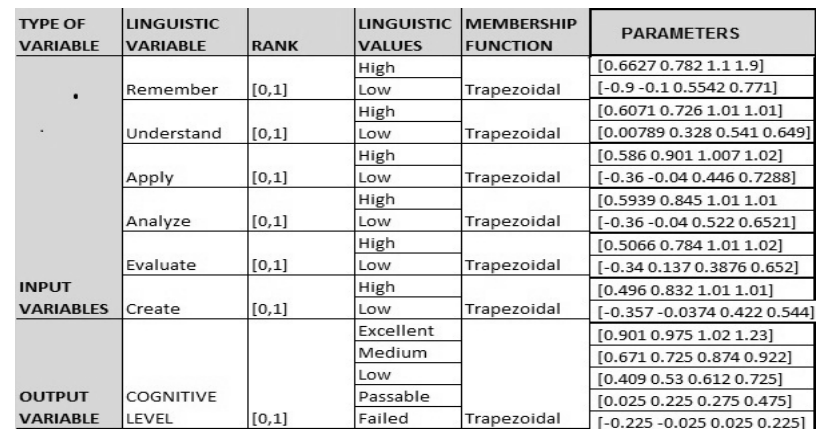

Fig. 2. Parameters of first Fuzzy Inference System of figure 1.

\section{$4 \quad$ Results}

The processes of figure 1 were simulated in a program with graphical user interfaces of matlab, version $2017 \mathrm{~b}$. In principle, for the purpose of testing, random values were considered for two rubrics with three problems, of each topic of algebra. Subsequently, process 1 is applied to obtain the values of Bloom's Taxonomy and the fuzzy value. The Figure 3 show the results of file in which, the process of simulate the assessment of 5 topics of unit 1 , when the counter " $i$ " of the figure 1 is equal to 1 , is accomplished. Figure 4 shows the results of the file that is responsible for performing the iterative process of the first diffuse cognitive map, which shows the values that the student must reach to get to understand all the topics of unit 1 . Finally, Figure 5 shows the process related to decision making related to the type of problems that the student must solve, according to the values obtained in the previous processes.

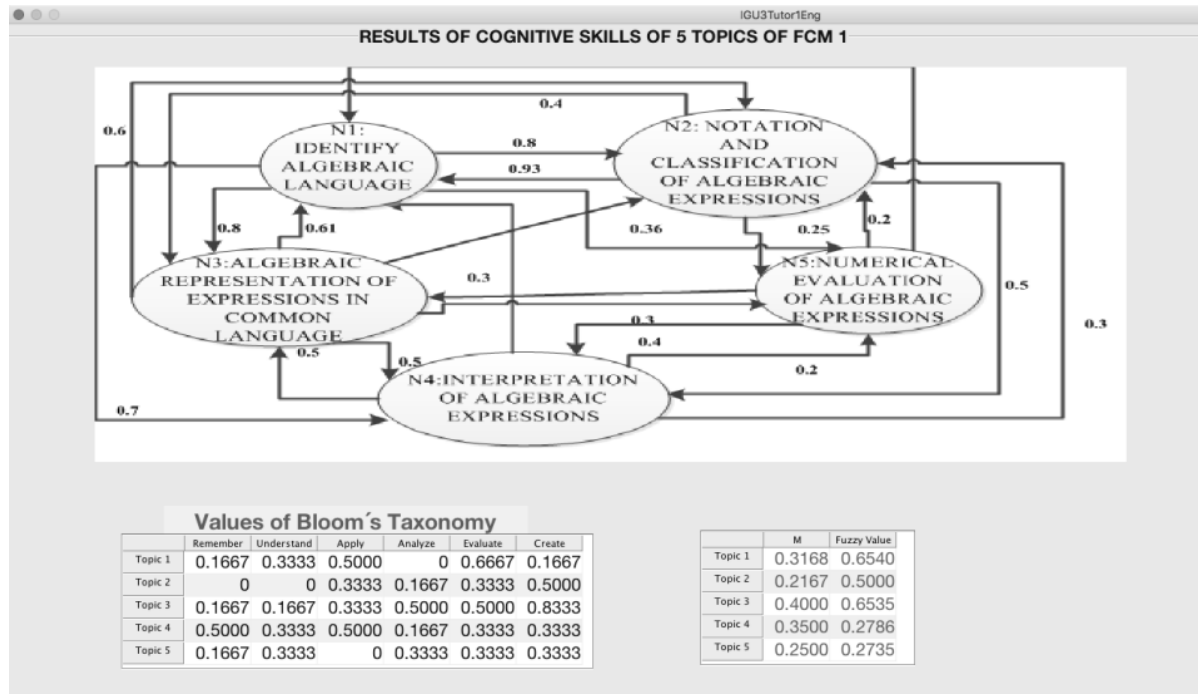

Fig. 3. Evaluation of the 5 topics of the FCM of unit 1. 


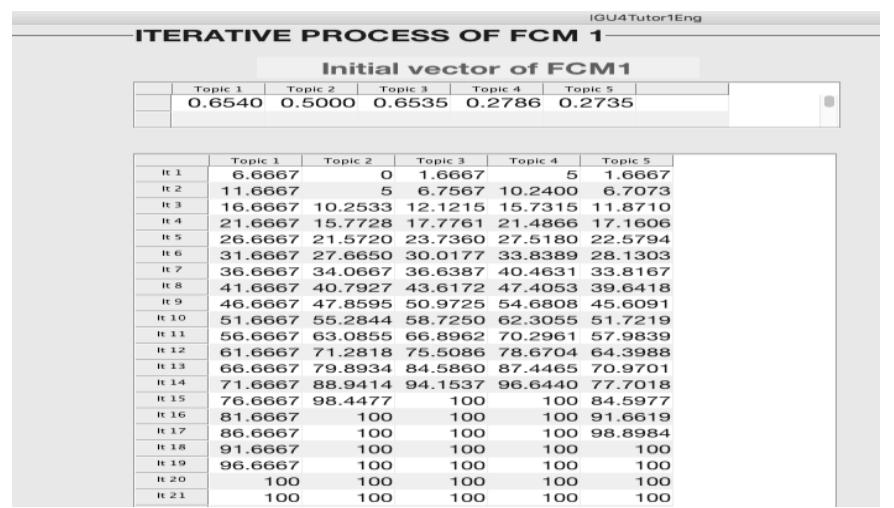

Fig. 4. Results of the iterative process of FCM 1.

\subsection{Analysis of Results}

In Figure 3, fuzzy values and average values are show, the difference among these values is that, the fuzzy value is representing of best manner, the characteristics of the student, in relation with your performance in each level of the Bloom's taxonomy. By example for topic 1, the average value is very low, even though the student performs well at the "evaluate" level of the Blooms taxonomy, which is why the fuzzy value is high. The results of Figure 5 are the suggestions that the tutor module will make to the student, which depend on the values of the Bloom's taxonomy that the student obtains and the dependencies between topics, therefore, for the topic 5 in figure 5 , the suggestion is that the student must work with fully resolved examples because their cognitive level is low.

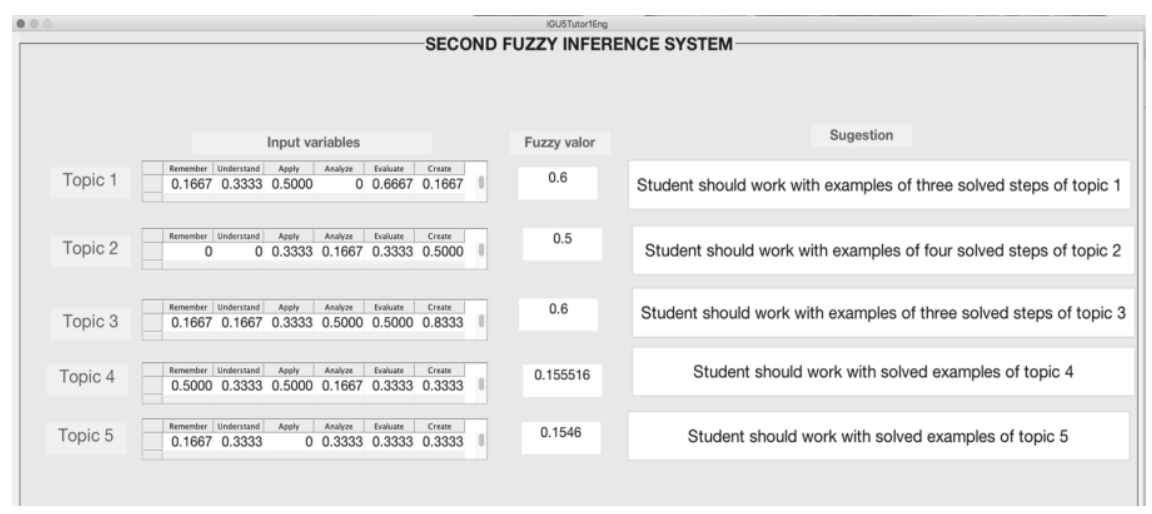

Fig 5. Application of the second Fuzzy Inference System for the tutor module.

\section{Conclusions}

The dependency between several algebra topics, as well as the relationship with problem solving, was modeled through of fuzzy models. It can be observed from the 
results shown that applying a variant of the original model proposed by Kosko, a model that converges into an optimal vector, using several incremental factors for each of the concepts related to the different subjects of algebra is obtained. This allows us to simulate the dynamic behavior of the students' learning process, and to determine the kind of problems that should be offered to them as exercises to enhance their learning.

The execution of the cognitive map convergence process has only been conducted to analyze the diverse combinations of input parameters. However, the goal is to obtain real data from several students that may serve as an input vector for map modeling and, thus, obtain the parameters that will help in the training of the model that will assign the problems and topics in which the student should work in. All this, in order to achieve the final objective, which is that the student can to solve completely and autonomously every of the problems in each topic, which will occur when the vectors associated to evaluation contain values that represent a total comprehension of every one of the concepts. This will be the vector to which all fuzzy cognitive maps should converge. This way, the efficiency of the domain module could be evaluated.

\section{References}

1. Chrysafiadi, K., Virvou, M.: A knowledge representation approach using fuzzy cognitive maps for better navigation support in an adaptive learning system. SpringerPlus 2(1), p. 81 (2013)

2. González, C., Mora, A., Toledo, P.: Gamification in intelligent tutoring systems. In: Proceedings of the Second International Conference on Technological Ecosystems for Enhancing Multiculturality - TEEM '14, pp. 221-225 (2014)

3. Groumpos, P.P.: Fuzzy Cognitive Maps: Basic theories and their application to complex systems. Fuzzy Cognitive Maps, 247, pp. 1-22 (2010)

4. Kalyuga, S.: Cognitive Load Theory: How Many Types of Load Does It Really Need? Educational Psychology Review 23(1), pp.1-19 (2011)

5. Kosko, B.: Fuzzy cognitive maps. International Journal of Man-Machine Studies 24(1), pp. 65-75 (1986a)

6. Papageorgiou, E.I., Salmeron, J.L.: Methods and Algorithms for Fuzzy Cognitive Mapbased Modeling. In: Papageorgiou, E.I. (ed.) Fuzzy Cognitive Maps for Applied Sciences and Engineering, pp. 1-28 (2014)

7. Rongmei, Z., Lingling, L.: Research on Internet Intelligent Tutoring System Based on MAS and CBR. In: 2009 International Forum on Information Technology and Applications, pp. 681-684 (2009)

8. Salden, R.J.C.M., Aleven, V., et al.: The expertise reversal effect and worked examples in tutored problem solving. Instructional Science 38(3), pp.289-307 (2010)

9. Skiba, D.J.: Bloom's digital taxonomy and word clouds. Nurs. Educ. Perspect. 34(4), pp. 277-280 (2013)

10. Sweta, S.. Lal, K.: Personalized Adaptive Learner Model in E-Learning System Using FCM and Fuzzy Inference System. International Journal of Fuzzy Systems (2017)

11. Ursini, S., Trigueros, M.: How do High School Students Interpret Parameters in Algebra? pp. 361-368 (2004) 